\title{
Osteogenic capacity of PLGA embedding simvastatin for biofunctionalization of titanium abutments
}

\author{
Raissa B. Curtarelli* DDs, MSc ${ }^{1}$ Mariane B. Sordi DDs, MSc ${ }^{1}$ Lenin I. Proaño \\ Minaca DDS1 César A. Magalhães Benfatti DDs, MSc, PhD1 Ricardo S. Magini \\ DDs, MSc, PhD1 Ariadne C. Cabral da Cruz DDs, MSc, PhD1
}

${ }^{1}$ Center of Education and Research in Dental Implants (CEPID), Federal University of Santa Catarina (UFSC). SC, Brazil

\section{Background}

Even though the high success rate of oral rehabilitations using dental implants, the peri-implant bone loss can still be observed. ${ }^{1}$ Biomaterials have been studied with incorporation of drugs that can be released locally and help the maintenance or the biological reestablishment of peri-implant tissues. ${ }^{2}$ To obtain a greater marginal stability, a titanium surface coating was proposed as a biofunctionalized prosthetic abutment.

\section{Aim}

This study evaluates the capacity of $0.6 \%$ simvastatin (SIM), incorporated into polylactic co-glycolic acid (PLGA) on titanium (Ti) surface, to promote osteogenic differentiation of stem cells of human exfoliated deciduous teeth (SHED).

\section{Methods and Materials}

Samples Preparation

Grade 4 Ti discs

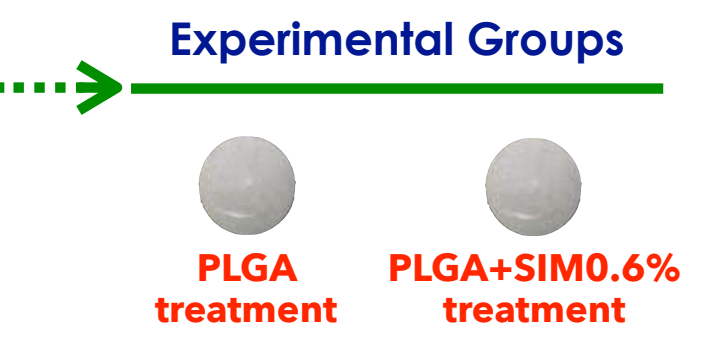

Treatment by immersion in PLGA or Treatment by immersion in PLGA or
PLGA+SIM0.6\% solution, dried by solvent evaporation technique.

Sterilization by ethylene oxide.

\section{Results}

SIM promoted the highest ALP activity at day $3(p<0.0001)$, while MC3T3-E1 group had the lowest. SHEDs with osteogenic and non-osteogenic medium and MC3T3-E1 demonstrated the lowest ALP activity at days 7 and 10 (Figure 1A). As shown in Figure 1B, Ti+PLGA+SIM0.6\% group showed the highest OCN expression at days 14 and $21(p<0.0001)$. Ti+PLGA group promoted the highest expression of ONC at day 21 ( $p=$ 0.0022). All control groups presented the lowest OCN expression at days 14 and 21. According to Figure 1C, SIM promoted the highest ONT expression at day $14(p<0.0001)$. The lowest ONT expressions were presented by SHED N-OST followed by SHED OST and MC3T3-E1 OST at days 14 and 21.
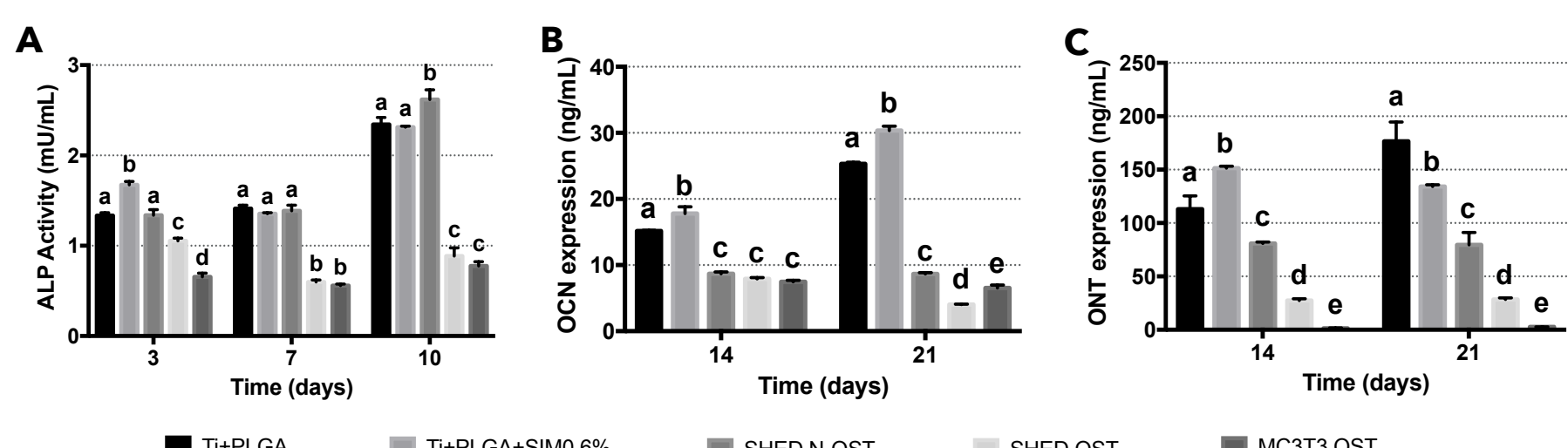

ETi+PLGA

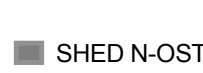

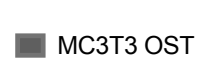

Figure 1. Quantification of ALP activity and bone proteins expression. A) SIM treatment promoted the highest ALP activity at day 3; B) The highest OCN expression were observed for Ti+PLGA+SIM0.6\% at days 14 and 21 ; C) SIM treatment promoted the highest ONT expression at day 3 . Results were expressed as mean $\pm S D$. Data were compared among the groups at experimental times independently. Different letters represent significant difference among groups $(p<0.05)$. ANOVA with Tukey's post hoc test.

\section{Conclusions}

Since SIM promoted the highest expressions of bone proteins and ALP activity at the most important experimental time, it can be concluded that biofunctionalization of Ti surface coated by PLGA incorporating SIM stimulated osteogenic differentiation of mesenchymal stem cells and can be considered as a clinical option. In vivo and clinical future experiments must be carry out to confirm this clinical indication.

\section{References}

\section{Osteogenic Differentiation Analysis}

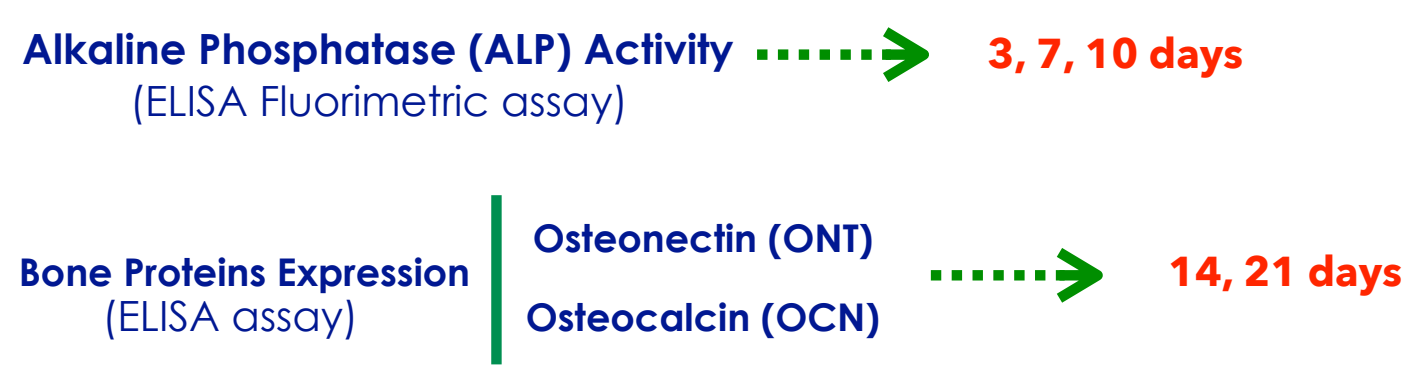

1. TABASSUM, A. et al. Evaluation of primary and secondary stability of titanium implants using different surgical techniques. Clinical Oral Implants Research, v. 25, n. 4, p. 487-492, 2014.

2. SANZ-SÁNCHEZ, I. et al. Biological effect of the abutment material on the stability of periimplant marginal bone levels: A systematic review and meta-analysis. Clinical Oral Implants Research, n. May, p. 1-21, 2018.

This study was partially funded by the International Team for Implantology (ITI) and Higher Education Personnel Improvement Coordination (CAPES). 